ISSN 1991-8631

Original Paper

http://indexmedicus.afro.who.int

\title{
Notification des effets indésirables de la spiruline chez l'homme : revus systématique
}

\author{
Mamoudou BARRY ${ }^{1,4^{*}}$, Moustapha OUEDRAOGO ${ }^{1,2,4}$, Moussa OUEDRAOGO ${ }^{1,2,4}$, \\ Seydou SOURABIE ${ }^{3}$, Inocent Pierre GUISSOU ${ }^{2,4,5}$, Bedouch PIERRICK ${ }^{6}$ et \\ Jean CALOP ${ }^{6}$ \\ ${ }^{1}$ Centre Hospitalier Pédiatrique Charles De Gaulle. \\ ${ }^{2}$ Unité de Formation et de Recherches en Sciences de la Santé (UFR/SDS). \\ ${ }^{4}$ Institut de Recherche en Science de la Santé (IRSS). \\ ${ }^{3}$ Pharmacy Department, Grenoble University, Hospital \& UJF-Grenoble. \\ ${ }^{5}$ Centre Hospitalier Universitaire Yalgado Ouedraogo, \\ ${ }^{6}$ Ecole Doctorale de Santé, Université de Ouagadougou. \\ *Auteur correspondant ; E-mail : bmoudou@yahoo.fr; BP 1198 Ouagadougou BF 01, Tél : 0022670409640
}

\section{RESUME}

La spiruline est une algue utilisée pour ses effets nutritionnels et thérapeutiques. Cependant son innocuité et ses éventuelles interactions avec les médicaments semblent peu étudier. Le présent travail se propose de résumer les quelques étude et les rapports de pharmacovigilance de la spiruline chez l'homme. La méthode a consisté à l'interrogation des bases de données PubMed, OMS, Medline, base des données des universités de Grenoble, centre de pharmacovigilance de Grenoble et de Bordeaux. Seules les études effectuées chez l'homme avec la spiruline, publiées et ayant notifiées les effets indésirables ont été retenues. Les critères de jugements ont porté sur les paramètres anthropométriques, biologiques, cliniques et les données des protocoles. Trois essais cliniques, trois études de cas cliniques et trente un rapports de pharmacovigilance dans le monde de 1996 à 2009 ont été retenus pour l'étude. Dans trois études randomisées contrôlées par un placebo, des décès ainsi que des perdues de vue dans le bras de la spiruline ont été notés. Les cas cliniques et les rapports de pharmacovigilance ont notifié des effets indésirables au niveau hépatique, dermatologique, digestif, des troubles hématologiques, rénaux et électrolytiques. Les effets indésirables liés au foie ont occupé la première place.

(C) 2013 International Formulae Group. All rights reserved.

Mots clés : Notification, Effets indésirables, Spiruline.

\section{INTRODUCTION}

La spiruline ou spirulina platensis est une cyanobacteries. Elle est riche en protéines, en lipides totaux, en glucides, en vitamines, en minéraux, en oligoéléments, et en pigments. Elle est consommée depuis l'antiquité. Plusieurs études ont démontré que la spiruline peut être utilisée pour lutter contre la famine et la malnutrition dans le monde (Karkos, 2011; Sall et al., 1999; Kulshreshtha et al., 2008). Les études Chez l'homme ont confirmé certains de ces effets thérapeutiques tels que les effets dans les maladies cardiovasculaires (Park et al., 2003), 
immun modulateurs, anti-inflammatoires (Deng et al., 2010), antianémiques (Selmi et al., 2011), et dans la récupération nutritionnelle (Branger et al, 2003; Simporé et al., 2006 ; Sall et al., 1999 ; Yamani et al., 2009 ; Marcel et al., 2011). Si la majorité des études ont eu pour objectif de mettre en évidence les effets bénéfiques de la spiruline, une minorité a notifié les effets indésirables rencontrés (Mazokopakis et al., 2008; Kraigher et al., 2008 ; Iwasa et al., 2002 ; Marles et al., 2011). Ces quelques études ont notifié des effets indésirables au niveau hépatique, dermatologique, digestif, des troubles hématologiques, rénaux et électrolytiques. La présente étude se propose de faire le point sur ces notifications des effets indésirables de la spiruline chez homme.

\section{MATERIALS AND METHODS}

La méthode a consisté à l'interrogation des bases de données Pubmed, OMS, Medline, base des données des universités de Grenoble, centre de pharmacovigilance de Grenoble et de Bordeaux. Seules les études effectuées chez l'homme avec la spiruline, publiées et ayant notifiées les effets indésirables ont été retenues. Les critères de jugements ont porté sur les paramètres anthropométriques, biologiques, les données cliniques et les données des protocoles.

\section{Les variables de l'étude}

Les signes cliniques, le nombre de décès, les perdus de vue ont été retenues pour apprécier les effets indésirables au cours des essais cliniques. Les déviations des paramètres biologiques par rapport aux valeurs normales, ont été retenues pour apprécier la toxicité dans les études de cas cliniques. Les manifestations cliniques ont été retenues pour les notifications dans les rapports de pharmacovigilance.

\section{Période}

Les études sur la spiruline chez l'homme, conduites jusqu'à juin 2012, publiées et retrouvées ont été retenues pour l'étude.

\section{RESULTATS \\ Notification des Effets Indésirables dans les trois études cliniques randomisées, contrôlées par un placebo}

Trois études cliniques randomisées, contrôlées par un placebo ont notifié les perdus de vue.

Dans le bras spiruline, Branger et al. (2003) dans son étude a enregistré 2,45\% de perdus de vue, Yamani et al. (2009) en a enregistré 7,6\% et Marcel et al. (2011) 35\%. Ce dernier seul sur les trois a notifié les effets indésirables à types de tuberculose pulmonaire (Tableau 1). Un auteur (Yamani, et al., 2009) sur les trois a notifié les décès $(10 \%$ dont $3,75 \%$ étaient issus du bras spiruline) (Tableau 1).

Notification des effets indésirables de la spiruline dans les études cas cliniques

Les trois cas cliniques, ont notifié des effets indésirables de la spiruline à type de rhabdomyolyse (un cas), de dermatoses (un cas) et hepatotoxicité (un cas) (Tableau 2).

Effets indésirables de la spiruline dans les rapports des centres de pharmacovigilance

La pharmacopée des Etats Unis d'Amérique (Marles et al., 2011), afin d'enregistrer la spiruline comme monographie, a rassemblé les déclarations des effets indésirables de la spiruline depuis 10 ans dans le monde entier à travers les centres de pharmacovigilance. Au total trente un (31) rapports ont été examinés (Tableaux 3 et 4). Le rapport MedWatch des USA a rapporté 11 cas d'effets indésirables, celui du Canada (The Canada Vigilance Program (CVP) 8; celui d'Australie 2 cas. Le centre de pharmacovigilance de l'OMS (l'Observatoire d'Uppsala (UMC) en a rapporté 10 cas. La Spiruline spp a été retenue avoir causé ses effets indésirables.

En résumant les effets indésirables par pathologie, ceux en rapport avec le foie viennent en première position et ceux dermatologiques occupent une deuxième place très importante (Tableau 5). 
M. BARRY et al. / Int. J. Biol. Chem. Sci. 7(4): 1568-1578, 2013

Tableau 1 : Notification des perdus de vue, des décès et effets Indésirables des trois études cliniques.

\begin{tabular}{|c|c|c|c|c|c|c|c|c|}
\hline \multirow[t]{2}{*}{ Auteurs } & \multirow[t]{2}{*}{ Traitement } & \multirow[t]{2}{*}{ Ethique } & \multirow[t]{2}{*}{ Randomisation } & \multicolumn{2}{|c|}{ Echantillon } & \multirow{2}{*}{$\begin{array}{l}\text { Perdus } \\
\text { de vues }\end{array}$} & \multirow[t]{2}{*}{ Décédés } & \multirow{2}{*}{$\begin{array}{c}\text { Effets } \\
\text { Indésirables }\end{array}$} \\
\hline & & & & $\begin{array}{l}\text { Echantillon } \\
\text { départ }\end{array}$ & $\begin{array}{c}\text { Echantillon } \\
\text { Arrivée }\end{array}$ & & & \\
\hline \multirow{4}{*}{$\begin{array}{l}\text { Branger et al., } \\
2003\end{array}$} & \multirow{4}{*}{ Non précisé } & \multirow{4}{*}{ NP } & G 1: aucun & 60 & 54 & 3 & NP & NP \\
\hline & & & G 2 : SP & 61 & 56 & 2 & $\mathrm{NP}$ & NP \\
\hline & & & $\mathrm{G} 3: \mathrm{SP}+\mathrm{P}$ & 61 & 55 & 1 & $\mathrm{NP}$ & NP \\
\hline & & & Total & 182 & 165 & 6 & 11 & \\
\hline \multirow{3}{*}{$\begin{array}{l}\text { Yamani et al., } \\
2009\end{array}$} & \multirow[t]{3}{*}{ Non précisé } & \multirow{3}{*}{$\begin{array}{c}\text { Ministère de la santé et l'approbation } \\
\text { du comité scientifique de la faculté } \\
\text { des sciences }\end{array}$} & G1 : Spiruline & 79 & 67 & 6 & 6 & \multirow{3}{*}{$\begin{array}{l}\mathrm{NP} \\
\mathrm{NP}\end{array}$} \\
\hline & & & G2 : Placebo & 81 & 61 & 10 & 10 & \\
\hline & & & Total & 160 & 128 & 16 & 16 & \\
\hline \multirow{3}{*}{$\begin{array}{l}\text { Marcel et al., } \\
2011\end{array}$} & \multirow{3}{*}{$\begin{array}{l}\text { ZDV, D4T et } \\
\text { 3TC }\end{array}$} & \multirow{3}{*}{$\begin{array}{l}\text { Comité national d'éthique du } \\
\text { Cameroun }\end{array}$} & G1 : Spiruline & 17 & 11 & 6 & NP & \multirow{3}{*}{$\begin{array}{l}\text { Tuberculose } \\
\text { pulmonaire, }\end{array}$} \\
\hline & & & G2 : Soja & 16 & 16 & 0 & NP & \\
\hline & & & Total & 33 & 27 & 6 & $\mathrm{NP}$ & \\
\hline
\end{tabular}


Tableau 2 : Effets indésirables de la spiruline des cas cliniques.

\begin{tabular}{|c|c|c|c|c|c|}
\hline Auteurs & $\begin{array}{l}\text { Type de } \\
\text { l'étude }\end{array}$ & Méthode & Résultats & Age/sexe & Posologie \\
\hline $\begin{array}{l}\text { Mazokopakis } \\
\text { et al., } 2008\end{array}$ & $\begin{array}{c}\text { Cas } \\
\text { Clinique }\end{array}$ & $\begin{array}{l}\text { Evaluation des taux } \\
\text { sériques de créatine } \\
\text { kinase (CK), } \\
\text { myoglobine sérique, } \\
\text { aldolase, transaminases } \\
\text { (ALAT / SGPT, AST / } \\
\text { SGOT) et de lactate } \\
\text { déshydrogénase (LDH) }\end{array}$ & 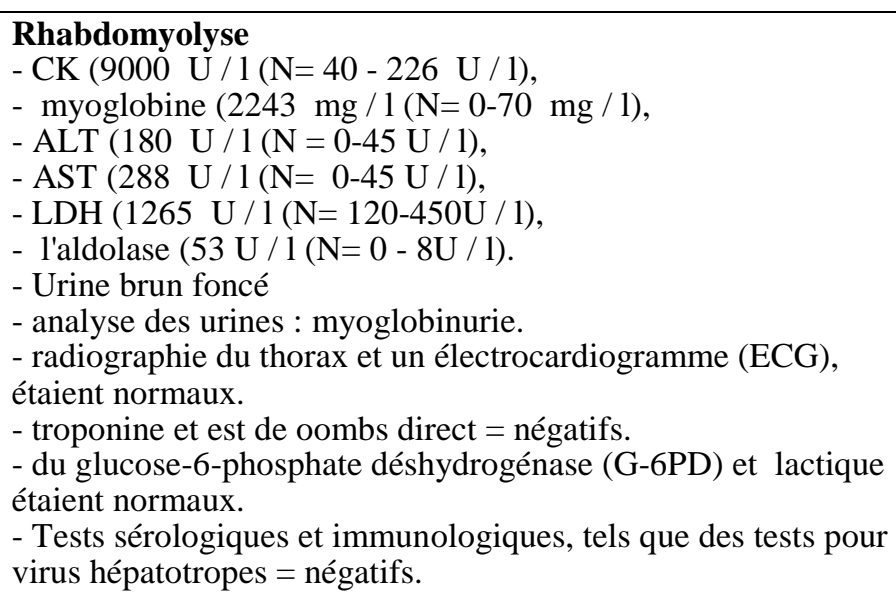 & $\begin{array}{l}28 \text { ans } \\
\text { sexe } \mathrm{M}\end{array}$ & $3 \mathrm{~g}$ par jour) \\
\hline $\begin{array}{l}\text { Kraigher et } \\
\text { al., } 2008\end{array}$ & $\begin{array}{c}\text { Cas } \\
\text { clinique }\end{array}$ & Clinique et biologiques & $\begin{array}{l}\text { Dermatoses } \\
\text { - signe de Nikolsky est positif } \\
\text { - bulle sous-épidermique } \\
\text { - Biopsie : une dermatite vésiculaire intra-et sous-cornéenne } \\
\text { avec acantholyse superficielle légère } \\
\text { - IgG positifs } \\
\text { - split test présence d' IgG, IgM, sur la face supérieure de la } \\
\text { bulle } \\
\text { - anticorps desmogléines } 1 \text { et } 3=\text { négatifs } \\
\text { - anticorps antinucléaires (ANA) = négatifs } \\
\text { - Amélioration après arrêt de la spiruline et traitement par la } \\
\text { néomycine et pommade à base de sulfadiazine d'argent }\end{array}$ & 82 ans/ F & NP \\
\hline
\end{tabular}


M. BARRY et al. / Int. J. Biol. Chem. Sci. 7(4): 1568-1578, 2013

\begin{tabular}{|c|c|c|c|c|}
\hline $\begin{array}{l}\text { Iwassa et al., } \\
2002\end{array}$ & Clinique et biologiques & 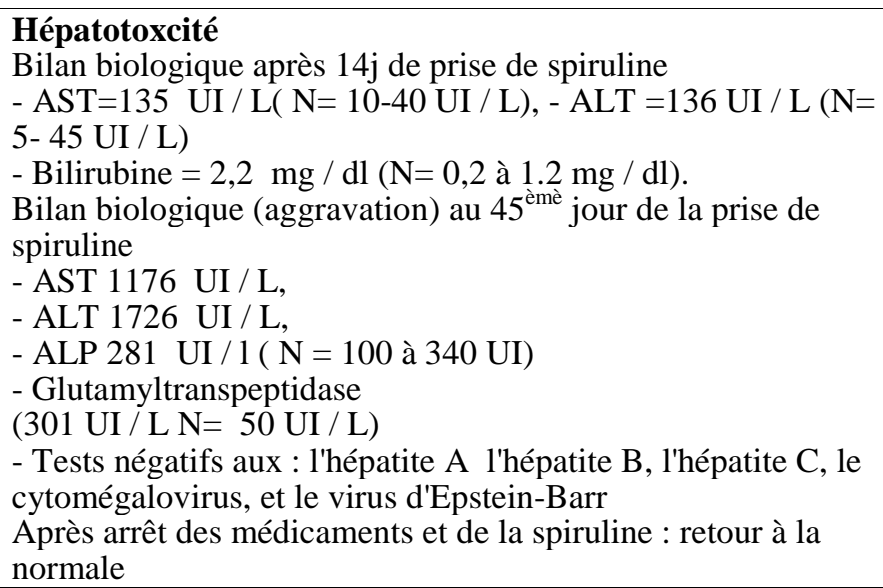 & $52 \mathrm{an} / \mathrm{H}$ & NP \\
\hline
\end{tabular}


Tableau 3: Rapports des événements indésirables de la spiruline de 1996 à 2003.

\begin{tabular}{|c|c|c|c|c|c|c|}
\hline Années & Continent & $\begin{array}{l}\text { âge et } \\
\text { sexe }\end{array}$ & Effets indésirable $s$ & Résultats & $\begin{array}{l}\text { Produits en } \\
\text { cause }\end{array}$ & Commentaires \\
\hline 1996 & Canada & $30 / \mathrm{F}$ & $\begin{array}{l}\text { Anomalies des tests hépatiques et } \\
\text { des malaises }\end{array}$ & & $\begin{array}{l}\text { Algues bleu- } \\
\text { vert }\end{array}$ & \\
\hline \multirow[t]{2}{*}{2000} & Canada & & cirrhose hépatique & & $\begin{array}{l}\text { Algues bleu- } \\
\text { vert }\end{array}$ & \\
\hline & Afrique & $62, \mathrm{~F}$ & Eruption & Récupéré & spiruline & $\begin{array}{l}\text { Effet disparu sur le retrait du } \\
\text { médicament; pas la reprise } \\
\text { du traitement }\end{array}$ \\
\hline \multirow[t]{3}{*}{2001} & USA & & $\begin{array}{l}\text { Élévation des valeurs des tests de la } \\
\text { fonction hépatique }\end{array}$ & & spiruline & \\
\hline & USA & & Accident ischémique transitoire & & spiruline & \\
\hline & $\overline{U S A}$ & & $\begin{array}{l}\text { la diarrhée, des vomissements, } \\
\text { tonico-cloniques convulsions, le } \\
\text { coma }\end{array}$ & & spiruline & \\
\hline \multirow[t]{4}{*}{2002} & USA & & $\begin{array}{l}\text { Élévation des valeurs des tests de la } \\
\text { fonction hépatique }\end{array}$ & & spiruline & \\
\hline & Canada & $36 / \mathrm{F}$ & L'œdème, éruption cutanée & & $\begin{array}{l}\text { Produit à base } \\
\text { de spiruline }\end{array}$ & \\
\hline & Canada & $37 / \mathrm{F}$ & Difficultés de dormir & $\begin{array}{l}\text { Récupéré } \\
\text { sans } \\
\text { complications }\end{array}$ & $\begin{array}{l}\text { Produits } \\
\text { contenant de } \\
\text { la spiruline }\end{array}$ & $\begin{array}{l}\text { Utilisation de plus de } 6 \\
\text { mois. }\end{array}$ \\
\hline & Australie & $37 / \mathrm{F}$ & Hémorragie & & Spiruline & \\
\hline
\end{tabular}


M. BARRY et al. / Int. J. Biol. Chem. Sci. 7(4): 1568-1578, 2013

\begin{tabular}{|c|c|c|c|c|c|c|}
\hline \multirow[t]{4}{*}{2003} & \multicolumn{2}{|l|}{ USA } & \multicolumn{2}{|l|}{$\begin{array}{l}\text { Hypercalcémie sévère, } \\
\text { déshydratation, la mort }\end{array}$} & \multicolumn{2}{|l|}{ Spiruline } \\
\hline & USA & & Sévère syndrome parkinsonien & & Spiruline & Fabricant inconnu \\
\hline & Australie & $51 / \mathrm{F}$ & $\begin{array}{l}\text { Malaise, douleurs abdominales, } \\
\text { douleurs à la poitrine, fièvre, } \\
\text { tachycardie, tachypnée, et des } \\
\text { vomissements }\end{array}$ & & spiruline & $\begin{array}{l}\text { pendant } 4 \\
\text { semaines }\end{array}$ \\
\hline & Afrique & $47, \mathrm{~F}$ & Diarrhée, des Crampes & Récupère & $\begin{array}{l}\text { Spiruline + } \\
\text { Eugynon }\end{array}$ & $\begin{array}{l}\text { Effet disparu après le } \\
\text { Retrait du médicament, } \\
\text { réaction réapparues Apres la } \\
\text { ré administration }\end{array}$ \\
\hline
\end{tabular}


M. BARRY et al. / Int. J. Biol. Chem. Sci. 7(4): 1568-1578, 2013

Tableau 4 : Rapports des événements indésirables de la spiruline de 2004 à 2008.

\begin{tabular}{|c|c|c|c|c|c|c|c|}
\hline Années & Continent & Posologie & $\begin{array}{l}\text { (âge et } \\
\text { sexe) }\end{array}$ & Effets indésirable (s) & Résultats & $\begin{array}{l}\text { Produits en } \\
\text { cause }\end{array}$ & Commentaires \\
\hline \multirow[t]{2}{*}{2004} & USA & & & $\begin{array}{c}\text { Élévation des enzymes } \\
\text { hépatiques, }\end{array}$ & $\begin{array}{l}\text { mort des } \\
\text { L'hépatite } \\
\text { toxique }\end{array}$ & $\begin{array}{l}\text { Plusieurs } \\
\text { Produits dont la } \\
\text { spiruline }\end{array}$ & $\begin{array}{l}\text { ATCD : Hépatite A, } \\
\text { B, C, d'alcool, la } \\
\text { jaunisse, et le } \\
\text { diabète }\end{array}$ \\
\hline & Canada & $\begin{array}{l}25 \mathrm{mg} \text { pendant } \\
3 \text { jours }\end{array}$ & $40 / \mathrm{F}$ & $\begin{array}{c}\text { L'insomnie, nausées, } \\
\text { vomissements; hypervigilance }\end{array}$ & & & $\begin{array}{l}\text { Utilisation d'un } \\
\text { produit contenant la } \\
\text { spiruline }\end{array}$ \\
\hline \multirow[t]{3}{*}{2005} & Australie & & $38 / \mathrm{F}$ & $\begin{array}{c}\text { Réaction de photosensibilité, } \\
\text { bouche sèche, douleurs } \\
\text { oculaires, des maux de tête }\end{array}$ & & $\begin{array}{l}\text { Multivitaminé } \\
\text { contenant de la } \\
\text { spiruline }\end{array}$ & \\
\hline & Suisse & Per Os $12 \mathrm{cp} / \mathrm{j}$ & $48, \mathrm{~F}$ & $\begin{array}{l}\text { Malaise et la fatigue } \\
\text { Prothrombine élevée }\end{array}$ & récupéré & $\begin{array}{l}\text { Spiruline }+ \\
\text { cenocoumarol }\end{array}$ & $\begin{array}{l}\text { apparition de la } \\
\text { réaction à partir de } 6 \\
\text { jours }\end{array}$ \\
\hline & Malaisie & $\begin{array}{l}\text { Per os } 500 \mathrm{mg} \\
\quad / \mathrm{j}(11 \mathrm{j})\end{array}$ & $15, \mathrm{M}$ & $\begin{array}{l}\text { Éruption Morbiliform; } \\
\text { pityriasis }\end{array}$ & $\begin{array}{c}\text { Pas } \\
\text { amélioration }\end{array}$ & Spiruline & \\
\hline 2007 & USA & & & L'hépatite toxique & & $\begin{array}{l}\text { Multivitaminé } \\
\text { contenant de la } \\
\text { spiruline }\end{array}$ & \\
\hline
\end{tabular}


M. BARRY et al. /Int. J. Biol. Chem. Sci. 7(4): 1568-1578, 2013

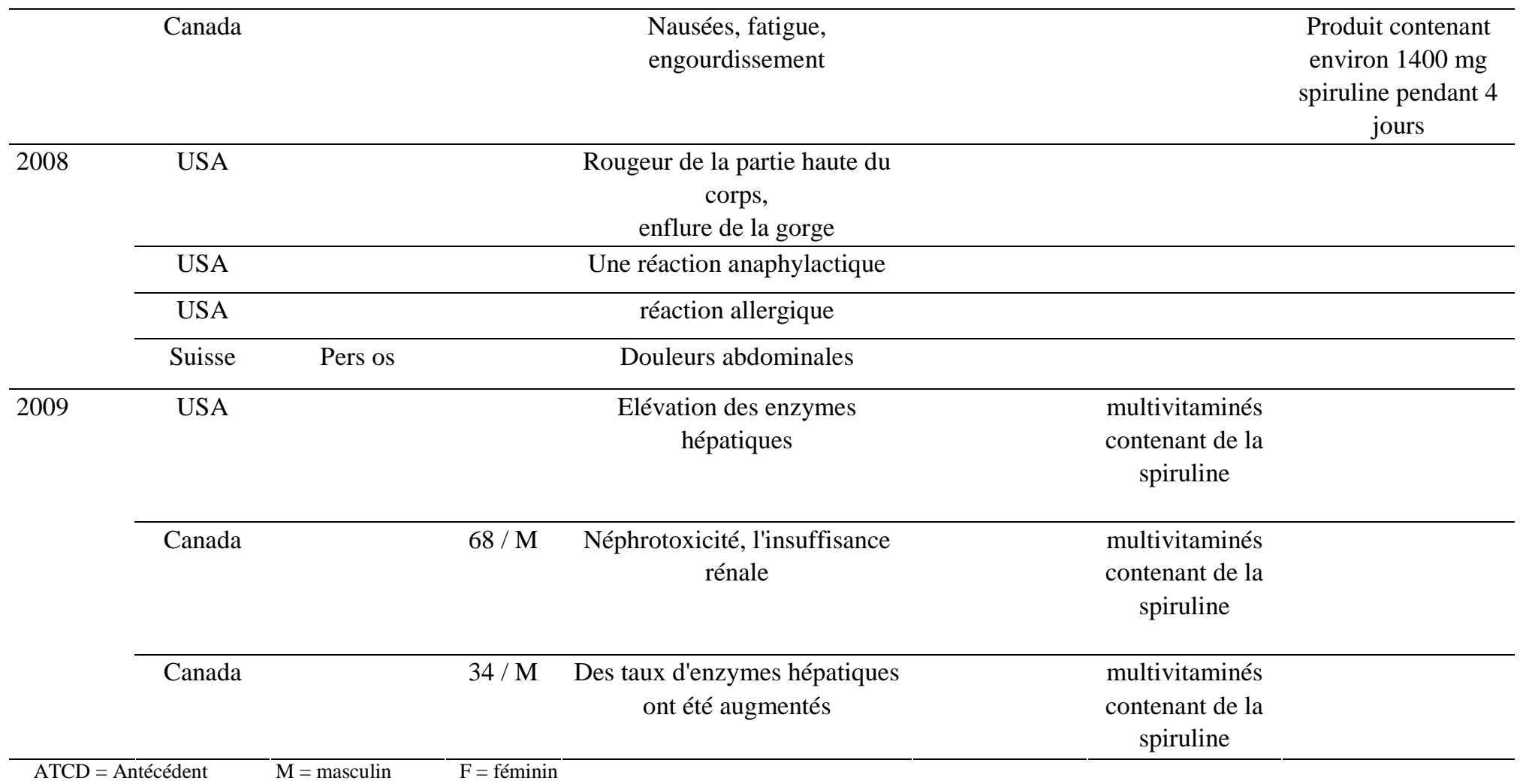


Tableau 5: Synthèse des effets indésirables de la spiruline rapportés en fonction des pathologies dans les centres de pharmacovigilance.

\begin{tabular}{lcc}
\hline Problèmes & Nombres de déclarations & \% \\
\hline Hépatiques & 12 & 34.30 \\
Dermatologiques & 7 & 20 \\
digestifs & 6 & 17.14 \\
SNC ? & 3 & 8.57 \\
Hématologiques & 3 & 8.57 \\
Néphrologiques & 1 & 2.85 \\
Electrolytiques & 3 & 8.57 \\
Total & 35 & 100 \\
\hline
\end{tabular}

SNC : Système Nerveux Central

\section{DISCUSSION}

Il n'ya pas eu une étude chez l'homme avec la spiruline dans le but d'étudier les effets indésirables. Aussi les études antérieures avec la spiruline chez l'homme n'ont pas notifié ces effets indésirables. Cependant, trois cas cliniques et des rapports de pharmacovigilance ont rapporté des effets indésirables de la spiruline surtout des effets hépatiques. Ces études ont montrée que la spiruline peut donner des effets indésirables ce qui nécessite une attention particulière pour son utilisation. Toute fois des études randomisées avec la spiruline chez l'homme seraient recommandé pour confirmer ou infirmer ces effets indésirables.

La tuberculose pulmonaire notée par Marcel et al. (2011) serait causée par l'état d'immunodépression des patients de son étude, infectés par le VIH. Cependant les perdus de vue des trois études, susciteraient des interrogations sur l'absence des effets indésirables notifié. Les décès notifiés par Yamani et al. (2009) ne peuvent incriminer la spiruline car les décès du groupe du placebo (10 décès) étaient supérieurs à ceux de la spiruline (6 décès).

La relation dose de spiruline et perdus de vu ou de décès dans les trois essais cliniques semble établie car Marcel et al. (2011) qui a administré $19 \mathrm{~g}$ par jour de spiruline a noté plus de perdus de vu $(18,18 \%)$ par rapport au 10\% noté par Yamani et al.
(2009) qui a utilisé $10 \mathrm{~g}$ par jour de spiruline, et 3,29\% de et Branger (2003) qui a utilisé $5 \mathrm{~g}$ par jour de spiruline.

Les transaminases élevées tant dans les cas cliniques que dans les rapports de pharmacovigilance, nécessiterait un suivi de ces paramètres lors de la prise de la spiruline et surtout dans l'association spiruline et les produits à métabolisme hépatique. Aussi l'étude du métabolisme de la spiruline ou des ses métabolites serait nécessaire pour les adaptations posologiques lors de son association avec les médicaments.

\section{Conclusion}

Les études cas cliniques ainsi que les rapports de pharmacovigilance notent des effets indésirables causés par la spiruline. Parmi ces effets indésirables, ceux hépatiques sont plus notés. La relation dose et perdus de vu est établie. Ces études ne sont qu'isolées à un cas ou à des rapports. Ce qui est difficile de généraliser que la spiruline donne des effets indésirables cependant une attention doit être observée lors de son utilisation. Une étude clinique randomisée de la spiruline et contrôlée par un placebo avec pour objectif la notification des effets indésirables, serait recommandée pour confirmer ou infirmer l'innocuité de la spiruline. 


\section{REMERCIEMENTS}

Nous remercions la Coopération Universitaire Franco - Burkinabé à travers sont projet RESEAU qui a soutenu ce travail en nous octroyant une bourse. Nous remercions le l'Université Joseph Fourier de Grenoble en particulier le personnel du service de la pharmacie clinique, du centre de pharmacovigilance et de la bibliothèque.

\section{REFERENCES}

Branger B, Cadudal JL, Delobel M, Ouoba H, Yameogo P, Ouedraogo D. 2003. Spirulina as a food supplement in case of infant malnutrition in Burkina-Faso. Arch. Pediatr., 10(5): 424-431.

Deng R, Chow TJ. 2010. Hypolipidemic, antioxidant, and antiinflammatory activities of microalgae Spirulina. Cardiovasc Ther., 28(4): e33-e45.

Iwasa M, Yamamoto M, Tanaka Y, Kaito M, Adachi Y. 2002. Spirulina-associated hepatotoxicity. Am. J. Gastroenterol., 97(12): 3212-3213.

Karkos PD, Leong SC, Karkos CD, Sivaji N, Assimakopoulos DA. 2011. Spirulina in clinical practice: evidence-based human applications. Evid Based Complement Alternat Med. doi: 10.1093/ecam/ nen058., 531053.

Kraigher O, Wohl Y, Gat A, Brenner S. 2008. A mixed immunoblistering disorder exhibiting features of bullous pemphigoid and pemphigus foliaceus associated with Spirulina algae intake. Int. J. Dermatol., 47(1): 61-63.

Kulshreshtha A, Zacharia AJ, Jarouliya U, Bhadauriya P, Prasad GB, Bisen PS. 2008. Spirulina in health care management. Curr. Pharm. Biotechnol., 9(5): 400-405.

Marcel AK, Ekali LG, Eugene S, Arnold OE, Sandrine ED, vonder W D. 2011. The Effect of Spirulina platensis versus Soybean on Insulin Resistance in HIVInfected Patients: A Randomized Pilot Study. Nutrients 18, 3(7): 712-724.

Marles RJ, Barrett ML, Barnes J, Chavez ML, Gardiner P, Ko R. 2011. United States pharmacopeia safety evaluation of spirulina. Crit Rev Food Sci Nutr., 51(7): 593-604.

Mazokopakis EE, Karefilakis CM, Tsartsalis AN, Milkas AN, Ganotakis ES. 2008. Acute rhabdomyolysis caused by Spirulina (Arthrospira platensis). Phytomedicine, 15(6-7): 525-527.

Park J., Kim WY. 2003. The effect of Spirulina on lipid metabolism, antioxidant capacity and immune function in Korean elderly. Korean J Nutr., 36: 287-297.

Sall M, Dankoko B, Badiane M. 1999. Results of a nutritional rehabilitation with spirulina in Dakar. Médecine d'Afrique Noire, 46(3) : 143-146.

Sall MG. 1999. la spiruline: une source alimentaire à promouvoir. Médecine d'Afrique Noire, 46(3): 140-142.

Selmi C, Leung PS, Fischer L, German B, Yang C-Y, Kenny TP. 2011.The effects of Spirulina on anemia and immune function in senior citizens. Cell. Mol. Immunol., 8(3): 248-254.

Simpore J, Kabore F, Zongo F, Dansou D, Bere A, Pignatelli S. 2006. Nutrition rehabilitation of undernourished children utilizing Spirulina and Misola. Nutr J., 5: 3.

Yamani E, Kaba-Mebri J, Mouala C, Gresenguet G, Rey JL. 2009. Use of spirulina supplement for nutritional management of HIV-infected patients: study in Bangui, Central African Republic. Med. Trop., 69(1): 66-70. 